\title{
Experimental model to evaluate the benefits of lutein to prevent retinal phototoxicity during pars plana vitrectomy surgery using xenon source light illumination in rabbits
}

\author{
Anderson Teixeira, Eduardo A. Novais ${ }^{*}[$, Emmerson Badaró, Acácio Lima, Michel Eid Farah \\ and Rubens Belfort Jr.
}

\begin{abstract}
Background: To evaluate the benefits of lutein in preventing retinal phototoxicity generated by xenon light sources during vitreoretinal surgery.

Methods: A prospective cross-sectional study in pigmented rabbit eyes exposed to different vitreoretinal surgery lighting simulations. Twenty Dutch-belted rabbits were divided into two groups exposed to two different xenon wavelength light sources filters ( $420 \mathrm{~nm}$ and $435 \mathrm{~nm}$ ). In addition, two subgroups were administered with daily supplemental of $10 \mathrm{mg}$ of Lutein systemically. Electroretinography (ERG), optical coherence tomography $(O C T)$ and fluorescein angiography (FA) were performed before and after surgery to quantify the retinal damage.

Results: All animals submitted to the experiment presented some degree of phototoxicity independent of wavelength light filter used. Retinal damage was evident as the FA presented areas of hyperfluorescence, and the OCT depicted increased reflective areas of the inner and outer retinal layers, and RPE. ERG showed a diffuse reduction of the a and $b$ waves amplitudes in all animals.
\end{abstract}

Conclusion: Use of systemic administration of lutein showed no benefit to avoiding retinal phototoxicity generate to xenon light source using filters of $435 \mathrm{~nm}$ and $420 \mathrm{~nm}$ when comparing to the control group.

Keywords: Lutein, Photo toxicity, Xenon light, Pars plana vitrectomy

\section{Background}

The eye is constantly exposed to sunlight and artificial light, which is essential for its biological functions such as vision and regulation of the circadian rhythm. However, when the exposure to light is excessive, radiation may generate visual impairment and even blindness [1].

In vitreoretinal surgery, the use of an intraocular illumination is necessary, increasing light exposure to the vitreous, retina and subretinal space [2]. Four types of

*Correspondence: eduardo@novais.md

Departamento de Oftalmologia, Universidade Federal de São Paulo,

Secretaria Administrativa, Rua Botucatu, 821, 10 andar, São Paulo, SP 04023-062, Brazil light sources are currently available: Metal halide, xenon, mercury, and halogen [3]. The use of light sources, however, can cause photochemical and thermal damage as an adverse effect to the retina [4]. The photochemical toxicity occurs when the eye is exposed to radiation light from the lowest visible wavelength (blue and violet), which becomes more harmful with wavelengths between 380 and $550 \mathrm{~nm}$ (Fig. 1) [5]. The thermal damage is caused by light sources with high-intesity irradiation/irradiance such as laser or xenon, causing the heating of tissues that absorb the radiation [6]. The retinal pigment epithelium (RPE) is prone to this type of injury due to the increased amount of pigment that can absorb light from different wavelengths. 


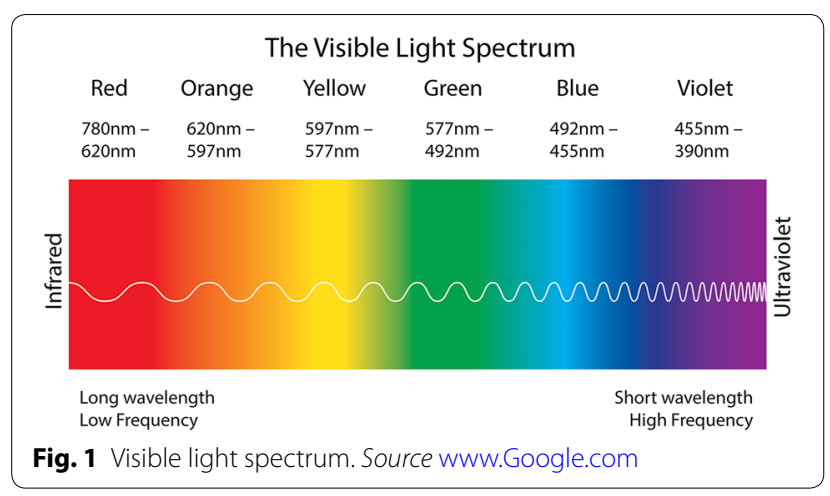

Lutein and Zeaxanthin are carotenoids present in the macular retinal pigment. A study in primates using microdensitometry observed a large pigment density in the axons of the photoreceptors (outer plexiform layer) in the foveal area and adjacent layers (inner and outer plexiform). The macular pigment also functions as a filter that absorbs short wavelengths of visible light, reducing chromatic aberration and dispersion in the. Filtration of short wave helps in preventing photochemical damage to cones and EPR [7-10].

In this study, we evaluated the benefits of supplemental lutein in preventing retinal phototoxicity generated by light sources for vitreoretinal surgery simulations in pigmented rabbits using two wavelengths $(420 \mathrm{~nm}$ and $435 \mathrm{~nm})$.

\section{Materials and methods}

The study was conducted in the Retina and Vitreous experimental laboratory of the Department of Ophthalmology of the Federal University of São Paulo. TwentyDutch belted rabbits weighing 1.5-2 kg were used according to the research standards of the Declaration of Helsinki and the Association for Research in Vision and Ophthalmology (ARVO) for experiments on animals.

A single experimental procedure was performed on the right eye of each rabbit. In total, 20 animals were divided into four groups (five animals for each group). They were divided into two groups exposed to different wavelengths (420 nm and $435 \mathrm{~nm}$ ) xenon lights (Xenon BrightStar illumination, DORC Inc, Netherlands) sources. The two wavelengths were chosen for this study as the $420 \mathrm{~nm}$ is the cut-off for core vitrectomy and general membrane removal, and $435 \mathrm{~nm}$ for removal of membranes adherent to the retina. In addition, two subgroups received supplemental $10 \mathrm{mg}$ of Lutein systemically mixed in the feed (LUT 10, Ophthalmos Inc, Brazil) and exposed to $420 \mathrm{~nm}$ or $435 \mathrm{~nm}$ wavelength. All rabbits were submitted to a macular surgery simulation during the experimental procedure, with the endoillumination placed near the retina with 30 min of light exposure. The maximum intensity of each of the light sources and endoillumination probe did not exceed the commercially used and no microscope light was used during the experiment.

\section{Data acquisition}

All rabbits were submitted to Electroretinography (ERG), optical coherence tomography (OCT) and fluorescein angiography (FA) preoperatively and seventh postoperative day.

\section{Anesthesia and preoperative preparation}

All surgical procedures and tests were performed under anesthesia after intramuscular injection of $35 \mathrm{mg} / \mathrm{kg}$ of ketamine hydrochloride (Phoenix Scientific Inc., USA) and $5 \mathrm{mg} / \mathrm{kg}$ of xylazine hydrochloride (Phoenix Scientific Inc., USA) in the cranial thigh (quadriceps) muscles. The pupil was dilated by instillation of cyclopentolate hydrochloride 1\% (Bausch \& Lomb Pharmaceuticals Inc., USA) and 5\% phenylephrine (Bausch \& Lomb Pharmaceuticals Inc., USA).

\section{Surgical technique}

The animals were covered with sterile drapes and an eyelid speculum placed, followed by instillation of a drop of $5 \%$ povidone-iodine. The following procedures were performed: 25 gauge sclerotomy for insertion of the light probe and placed on a fixed position using a micromanipulator. At the end of the procedure, the sclerotomy sight was sutured using 7-0 Vycril, and a sterile drop of antibiotics and steroids was applied. The rabbits were sacrificed by intravenous injection of $2 \mathrm{ml}$ of phenobarbital seven days after surgery.

\section{Electroretinography}

ERG was performed preoperatively and seven days after the procedure. Rabbits were kept in a dark room for one hour before the exam. Dark red illumination was used to handle the rabbits as they were anesthetized with an intramuscular injection of ketamine and xylazine. After dilation of the pupils, an electrode (Burian Allen, Hansen Ophthalmic, Iowa City, USA) covered with $1 \%$ methylcellulose was placed on the surface of the cornea; another similar electrode was placed on the temporal side as reference electrode; a gold dome of ground electrode in clip form, filled with electrolytic gel was placed in the animal's ear.

Dark-adapted ERG "a" (rod-mediated) and "b"-waves (mediated by bipolar and Müller cells) evoked at a luminance of $1.30 \log \mathrm{cd} \mathrm{s} / \mathrm{m} 2$ were recorded. The responses from five consecutive flashes generated an average to determine the dark adaptation responses. After light adaptation for at least $25 \mathrm{~min}$, light-adapted ERG was 
performed, and "a"-wave (cone-mediated) evoked at a luminance of $1.30 \log \mathrm{cd} \mathrm{s} / \mathrm{m} 2$ were recorded. The ERG followed the standard protocol recommended by ISCEV (International Society for Clinical Electrophysiology of Vision), in which five responses are recorded: (1) rods scotopic response; (2) maximum scotopic response (rods and cones); (3) scotopic response of oscillatory potentials; (4) photopic response of cones to single flash; (5) photopic flicker response to $30 \mathrm{~Hz}$. The parameters analyzed were the amplitudes of the responses, measured in microvolts $(\mu \mathrm{V})$ and the implicit time of b-wave, measured in milliseconds (ms). All values were compared with standard values using the same procedure standardized in our laboratory.

\section{Retinography}

The color fundus photography was performed using a specific fundus camera (TRC Topcon, Topcon, Tokyo, Japan) preoperatively and seven days after the procedure (depending on the subgroup).

\section{Fluorescein angiography (FA)}

FA was performed by intravenous injection of $0.3 \mathrm{ml}$ of sodium fluorescein 10\% (Ophthalmos, São Paulo, Brazil) in the auricular vein of the animal. FA was performed preoperatively and seven days after the procedure using HRA angiography (Heidelberg Inc, Germany).

\section{Optical coherence tomography (OCT)}

OCT was performed preoperatively and seven days after the procedure. This examination was conducted using the spectral-domain Spectralis OCT (Heidelberg Inc, Germany). After pupillary dilation, a $20 \times 15$-degree high-resolution 19 sections B-scan spaced by $120 \mu \mathrm{m}$ acquisition protocol was performed at the inferiorlly, inferotemporally and inferonasally to the optic nerve.

\section{Analysis of results}

The analysis of the results was carried out by qualitative and quantitative comparison of the preoperative and postoperative ERG, OCT and FA.

\section{Results}

All animals submitted to the experiment presented some degree of phototoxicity regardless of the wavelength filter and use of systemic lutein. Retinal damage was evident as the FA presented hyperfluorescence in the area of light exposure, and the OCT depicted increased reflective areas of the inner and outer retina layers, and RPE (Tables 1 and 2). We believe that the damage occurred due to the RPE absorption of the wavelength irradiance and possible increase in tissue temperature secondary

\begin{tabular}{llll}
$\begin{array}{l}\text { Table } \mathbf{1} \text { Rabbits } \\
\text { irradiance }\end{array}$ & exposed to & $\mathbf{4 2 0}-\mathbf{n m}$ filter wavelength \\
\hline Rabbits & Lesion seen on FA & Lesion seen on OCT & Lutein \\
\hline 1 & Yes & Yes & Yes \\
2 & Yes & Yes & Yes \\
3 & Yes & Yes & Yes \\
4 & Yes & Yes & Yes \\
5 & Yes & Yes & Yes \\
11 & Yes & Yes & No \\
12 & Yes & Yes & No \\
13 & Yes & Yes & No \\
14 & Yes & Yes & No \\
15 & Yes & Yes & No \\
\hline
\end{tabular}

light exposure (Figs. 2, 3, 4, 5, 6 and 7). The ERG examination showed a significant $a$ and b-waves amplitude decrease (Figs. 1, 2, 3 and 4).

The FA and OCT from the subgroup exposed to the two different wavelengths after systemic administration of lutein are presented on Fig. 2, 3, 4 and 5. The ERG from the same group is shown on Figs. 8 and 9.

The FA and OCT from the subgroup exposed to the two different wavelengths without systemic administration of lutein (control groups) are presented on Figs. 6 and 7. The ERG from the same group is shown on the Figs. 10 and 11.

\section{Discussion}

The modern pars plana vitrectomy surgery evolved with increasing gauge and speed of cutting tips. Another crucial factor for surgical success is the better illumination during the surgery, and decrease in tissue damage secondary to light exposure irradiation of the retina and EPR, offered by new light sources such as LED and Xenon [3]. However, improvement in visualization by these new light sources can be associated to greater retinal damage due to the photochemical toxicity of short wavelength (blue and violet). In addition, the RPE may absorb the light, generating heat, thus causing thermal damage. Also, smaller caliber probes can concentrate energy in a specific retina area, since the light is dissipated in proportion to the size of the endoillumination tip. A practical example is a 23-gauge probe, which irradiates a larger area than a 25-gauge probe, dissipating the amount of radiated energy in a larger area, thus, being less harmful.

The photochemical toxicity occurs when the eye is exposed to radiation from shorter wavelengths of visible light (blue and violet), which become more harmful with wavelengths between 380 and $550 \mathrm{~nm}$ [5]. The thermal damage is caused by light sources with 
Table 2 Rabbits exposed to 435-nm-wavelength irradiance

\begin{tabular}{llll}
\hline Rabbits & Lesion seen on FA & Lesion seen on OCT & Lutein \\
\hline 6 & Yes & Yes & Yes \\
7 & Yes & Yes & Yes \\
8 & Yes & Yes & Yes \\
9 & Yes & Yes & Yes \\
10 & Yes & Yes & Yes \\
16 & Yes & Yes & No \\
17 & Yes & Yes & No \\
18 & Yes & Yes & No \\
19 & Yes & Yes & No \\
20 & Yes & Yes & No \\
\hline
\end{tabular}

high-intensity irradiation/irradiance such as laser or xenon, causing the heating of tissues that absorb the radiation. This mechanism does not comply with the principle of reciprocity between the amount of radiation and the duration of exposure; it depends on the conduction of heat by the irradiated tissue. When the target is small, it will cool faster and further irradiation to cause thermal injury is necessary [6].

The macular pigment also functions as a filter that absorbs short wavelengths of visible light, reducing chromatic aberration and dispersion in the fovea. Filtration of short wave helps in preventing photochemical damage to cones and the RPE. The Lutein and Zeaxanthin are present in the photoreceptors axons, pigment in the fovea area and adjacent layers; and theoretically may also have an anti-inflammatory effect [7-15]. In this study, the $420 \mathrm{~nm}$ and $435 \mathrm{~nm}$-wavelengths generated damage in both neurosensory retina as well as in the RPE, supporting the literature concern about the use of lighting sources with a wavelength close to ultraviolet waves [16, 17].

In all studied subjects there was evidence of the presence of phototoxicity due to the irradiance of the light sources in simulate vitreoretinal macular surgery. The use of lutein as a protective factor does not prevent the onset of phototoxicity characteristic changes observed in FA and OCT. The anatomical lesions were well evidenced in the examination with FA as they presented as hyperfluorescence, and in OCT as increased reflective of the neurosensory retina and thickening of the line that corresponds to the retinal pigment epithelium. In summary, the FA and OCT findings showed that the EPR plays an important role in generating retinal damage from the thermal and photochemical point of view. All lesions were present in all the wavelengths filters as seen in the figures described in this paper.

The preliminary results shows that the model in pigmented rabbits were suitable for evaluation the retinal
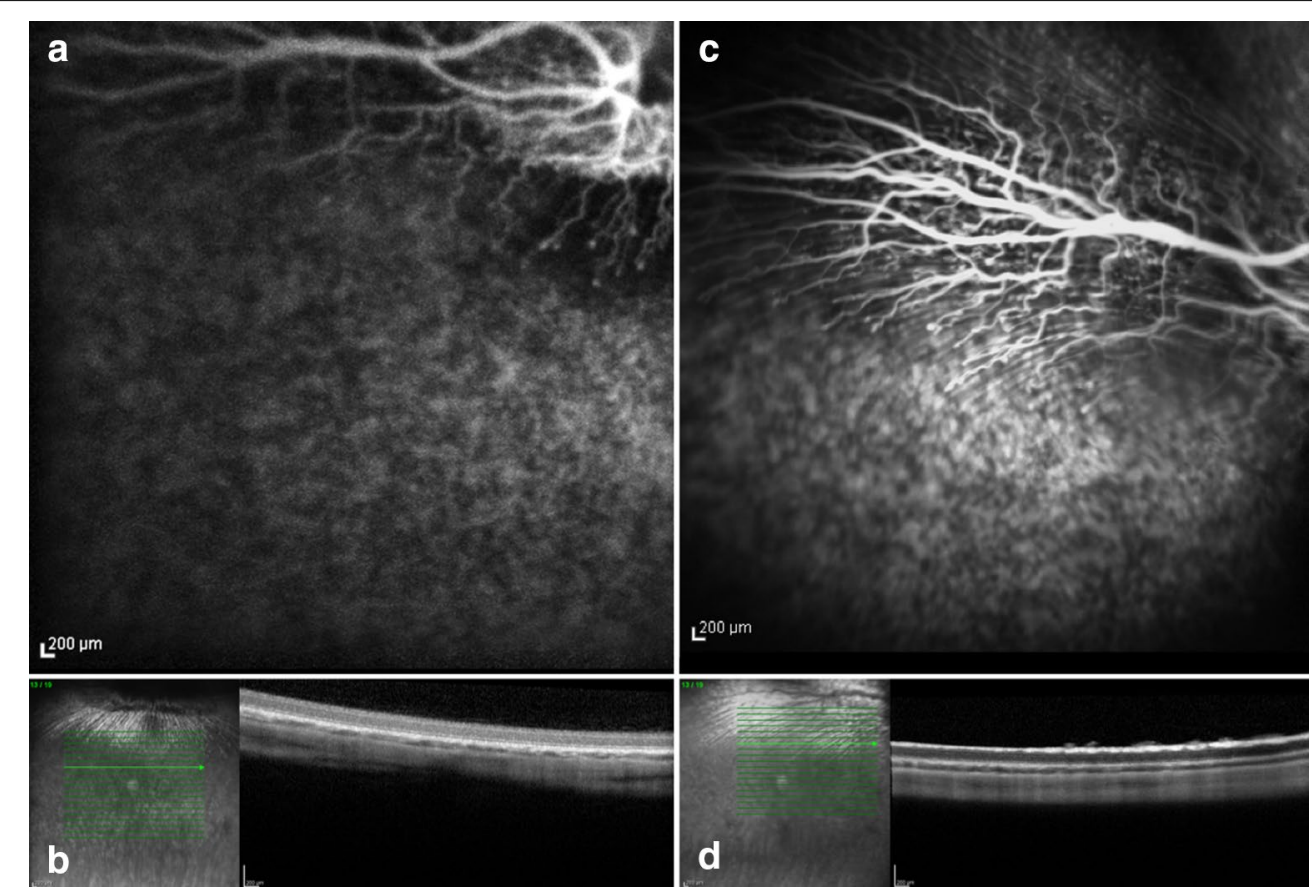

Fig. 2 Preoperative and seven days posoperative fluorescein angiography and OCT. Rabbit (no. 1) exposed to $420 \mathrm{~nm}$ illumination after systemic administration of lutein. Postoperative hyperfluorescence corresponds to the exposed area and increased reflective of the inner and outer layers of the retina seen on OCT 


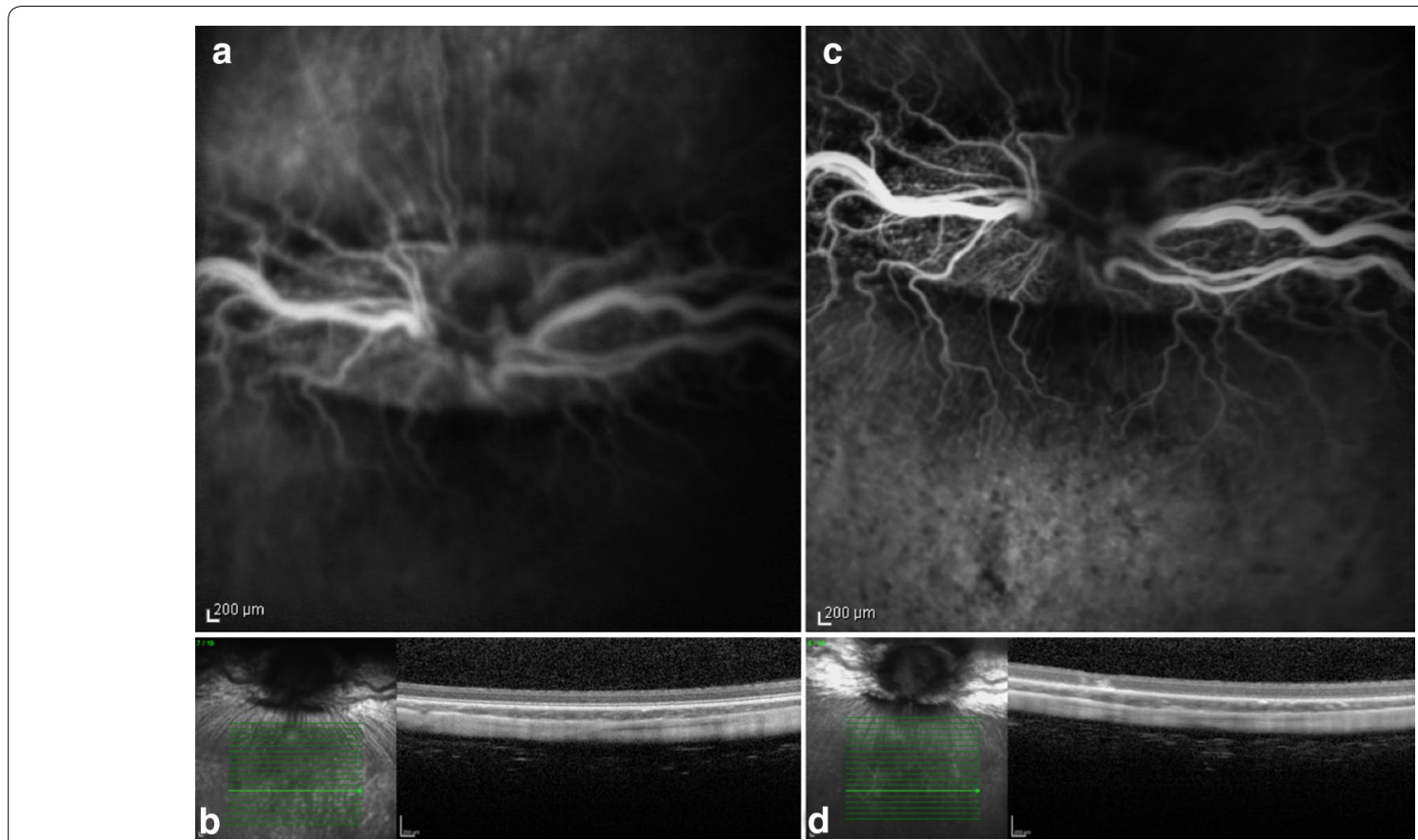

Fig. 3 Preoperative ( $\mathbf{a}$ and $\mathbf{b}$ ) and seven days postoperative (c and $\mathbf{d}$ ) fluorescein angiography and OCT. Rabbit (no. 2) exposed to light of $420 \mathrm{~nm}$ after systemic administration of lutein. Postoperative hyperfluorescence (c) corresponds to the exposed area and increased reflective of the inner and outer layers of the retinaseen on OCT (d)

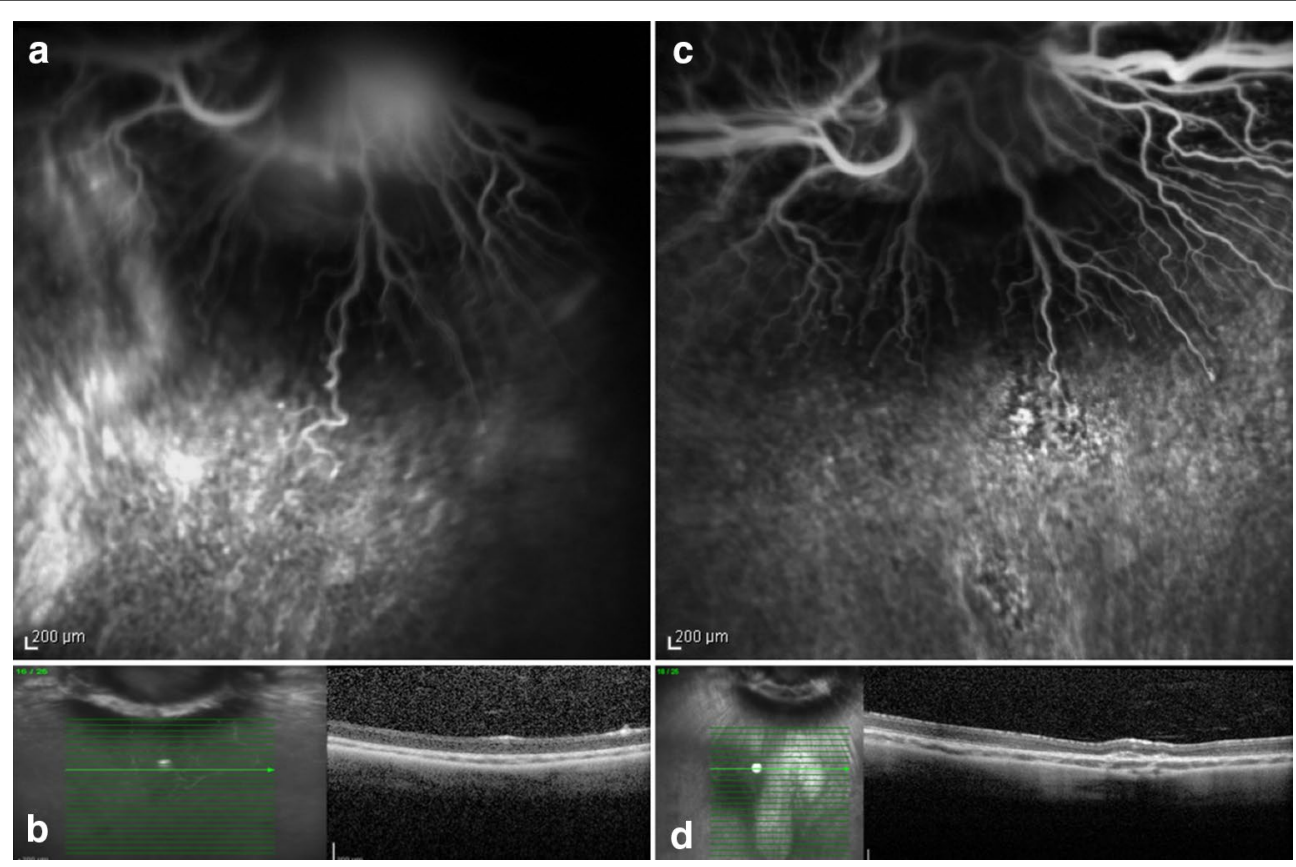

Fig. 4 Preoperative (a and $\mathbf{b}$ ) and seven days postoperative (c and $\mathbf{d}$ ) fluorescein angiography and OCT. Rabbit (no. 6) exposed to $435 \mathrm{~nm}$ illumination after systemic administration of lutein. Postoperative hyperfluorescence (c) corresponds to the exposed area and increased reflective of the inner and outer layers of the retina seen on OCT (d) 


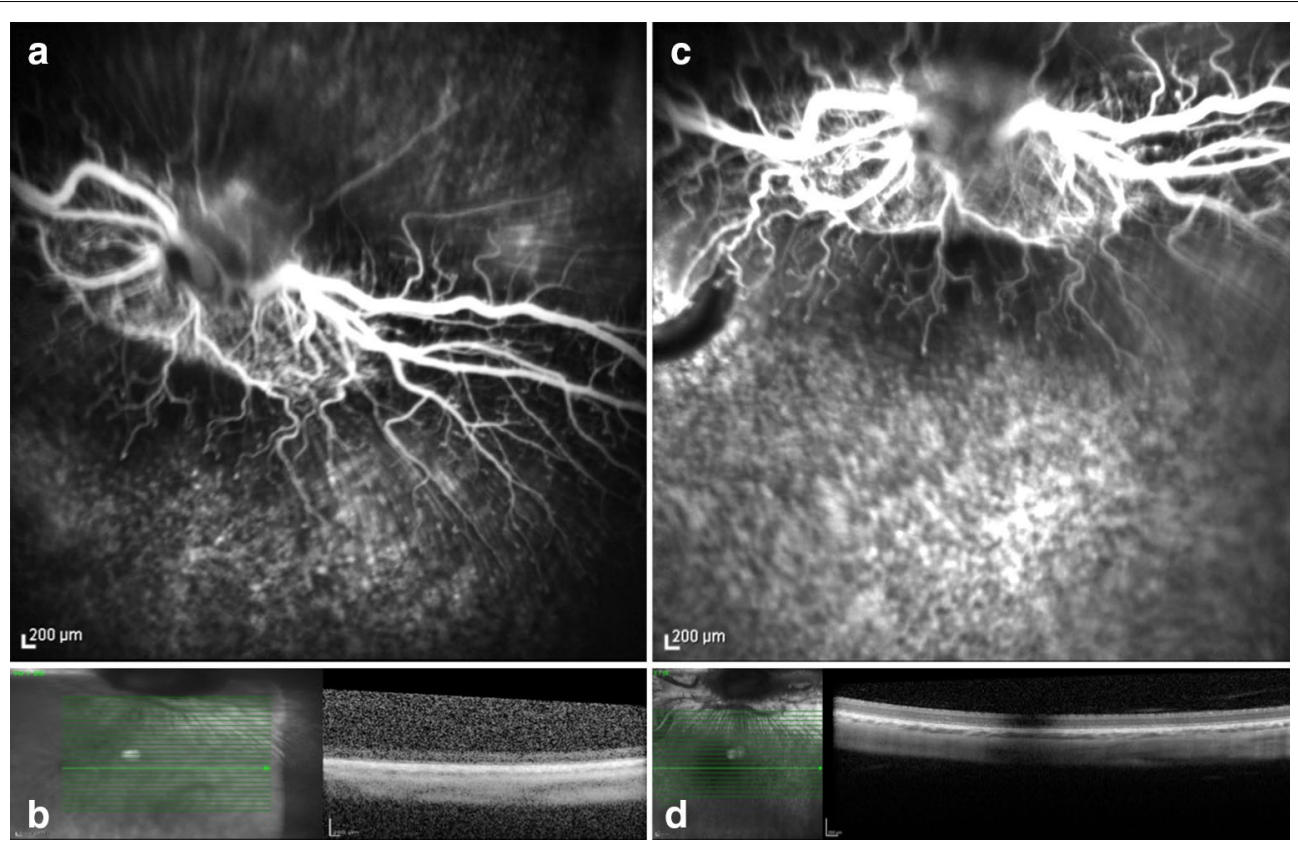

Fig. 5 Preoperative (a and b) and seven days postoperative (c and $\mathbf{d}$ ) fluorescein angiography and OCT. Rabbit (no. 8) exposed to $435 \mathrm{~nm}$ illumination after systemic administration of lutein. Postoperative hyperfluorescence (c) corresponds to the exposed area and increased reflective of the inner and outer layers of the retina seen on OCT (d)

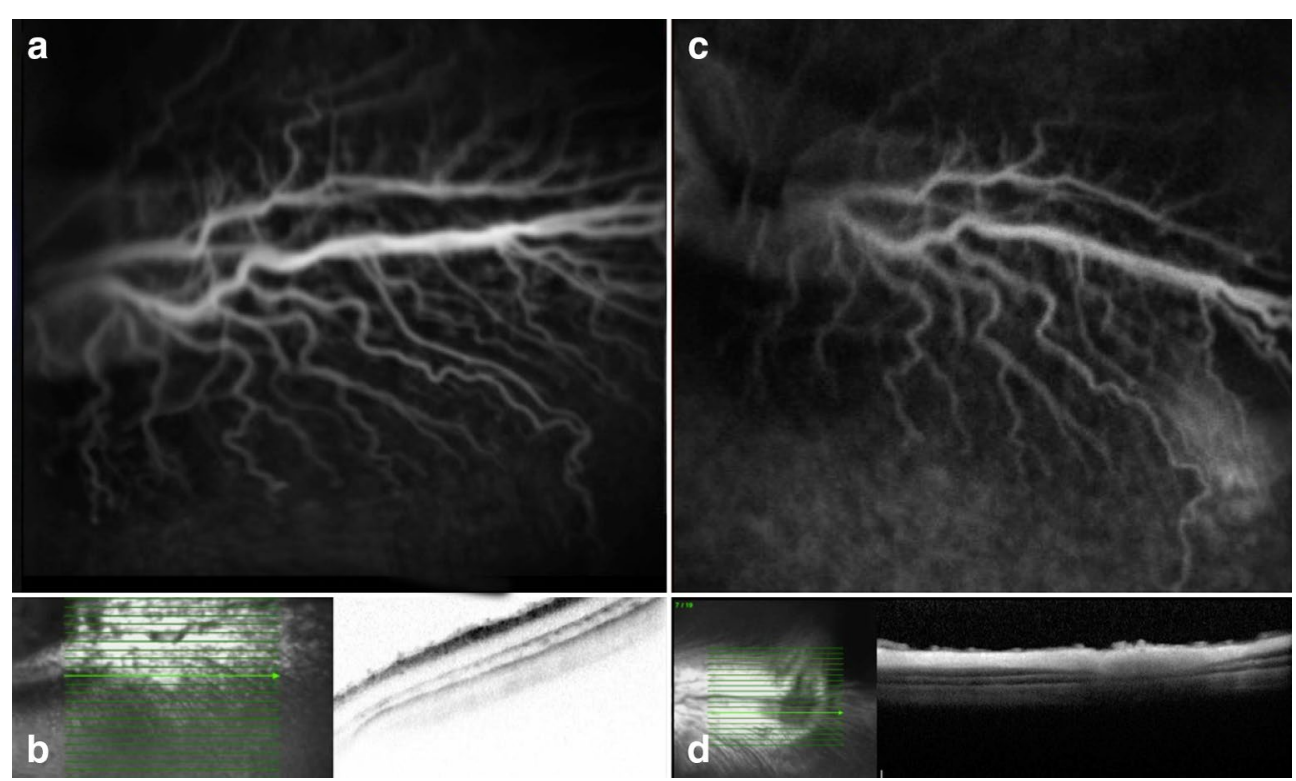

Fig. 6 Preoperative ( $\mathbf{a}$ and $\mathbf{b}$ ) and seven days postoperative (c and $\mathbf{d}$ ) fluorescein angiography and OCT. Rabbit (no. 12) exposed to light $420 \mathrm{~nm}$ without systemic administration of lutein. Postoperative hyperfluorescence (c) corresponds to the exposed area and increased reflective of the inner and outer layers of the retina seen on OCT (d) 


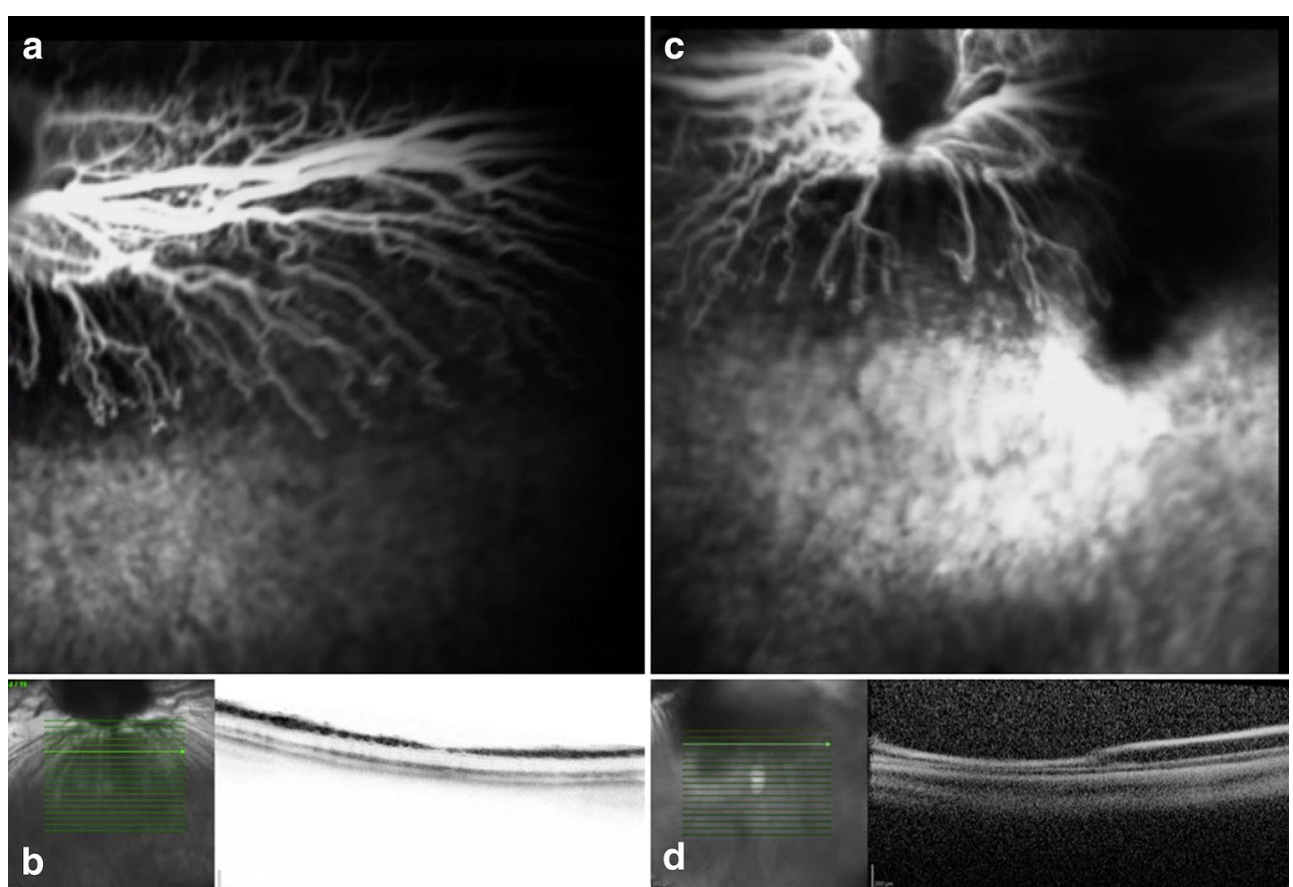

Fig. 7 Preoperative ( $\mathbf{a}$ and $\mathbf{b}$ ) and seven days postoperative (c and $\mathbf{d}$ ) fluorescein angiography and OCT. Rabbit (no.16) exposed to $435 \mathrm{~nm}$ illumination without the administration of lutein. Postoperative hyperfluorescence (c) corresponds to the exposed area and increased reflective of the inner and outer layers of the retina with tissue thinning evidenced by examining OCT (d)

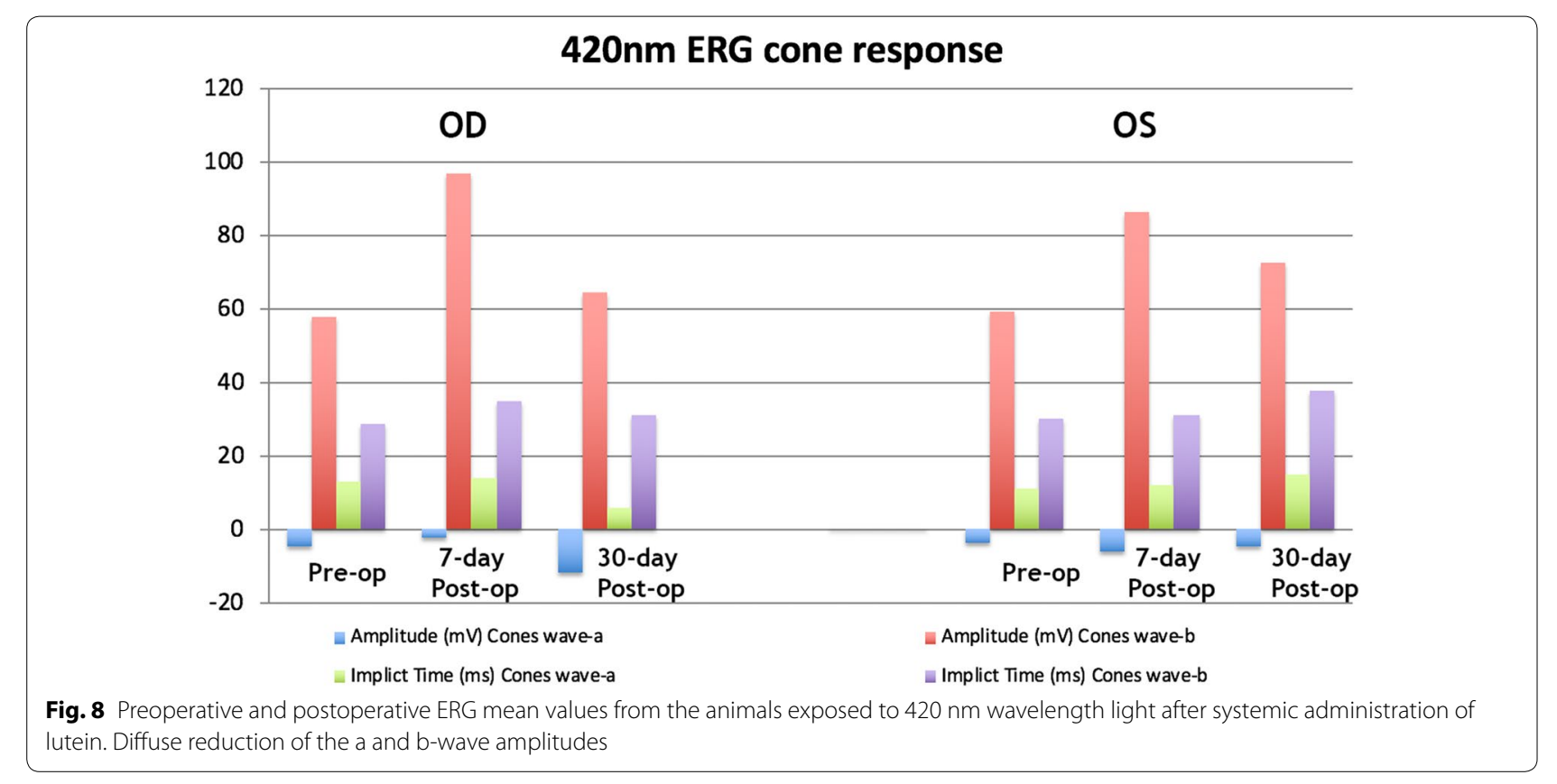

damage using FA examination and OCT. Factors such as exposure time, distance from the lighting, wavelength, illumination caliber, can be related to injuries and functional retinal changes.
This study has limitations due to the use of pigmented rabbits. The highly pigmented RPE absorbs the light and can increase tissue temperature, thus contributing to the increase of lesions presented in the results. 

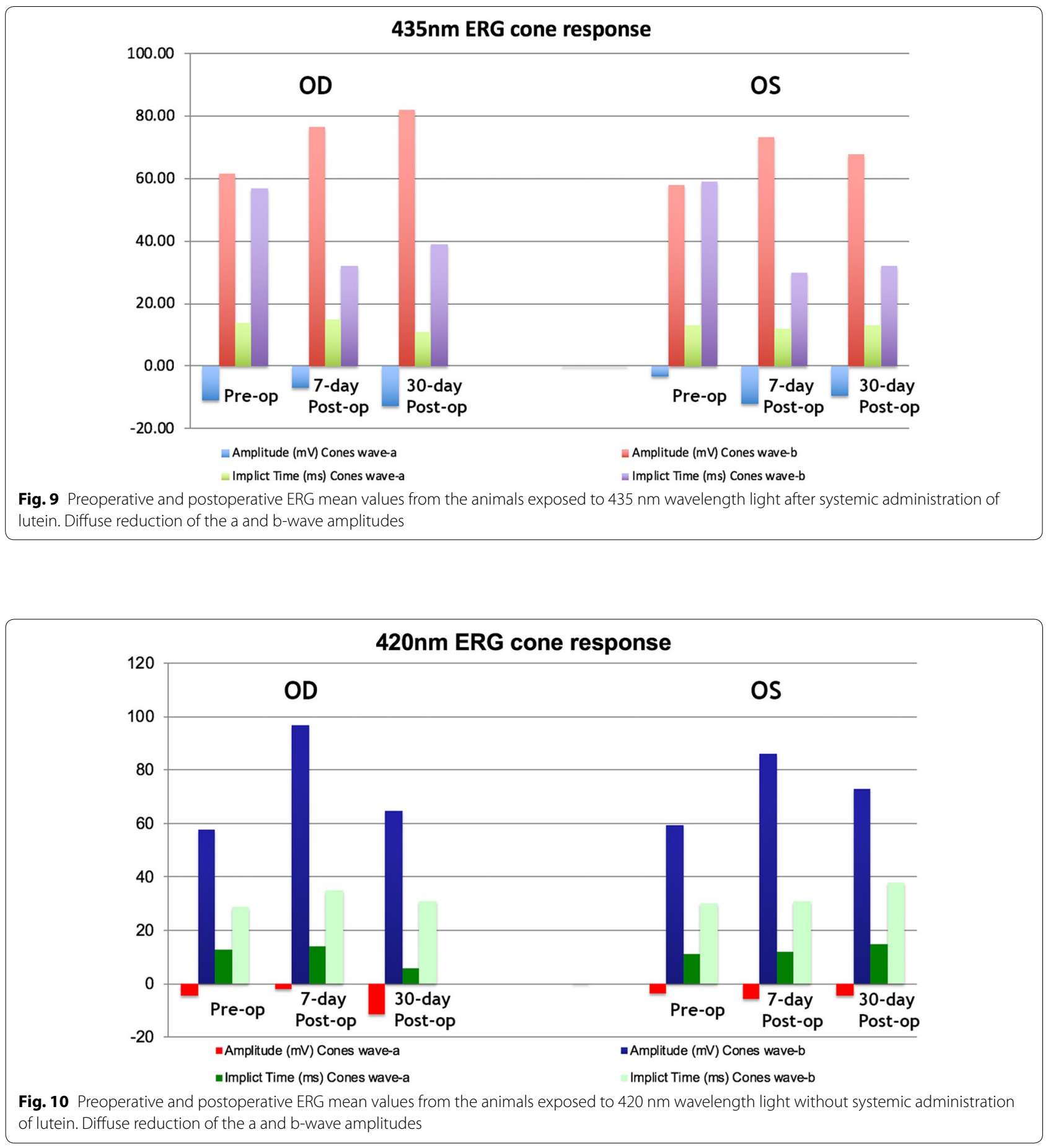

Future designs using albino rabbits are required to get more accurate results for the phototoxicity wavelength. Another limitation from this study is due to the OCT model used in the experiment, since the absence of "en face" image limits the quantitative assessment of the damage of each retina layer. 


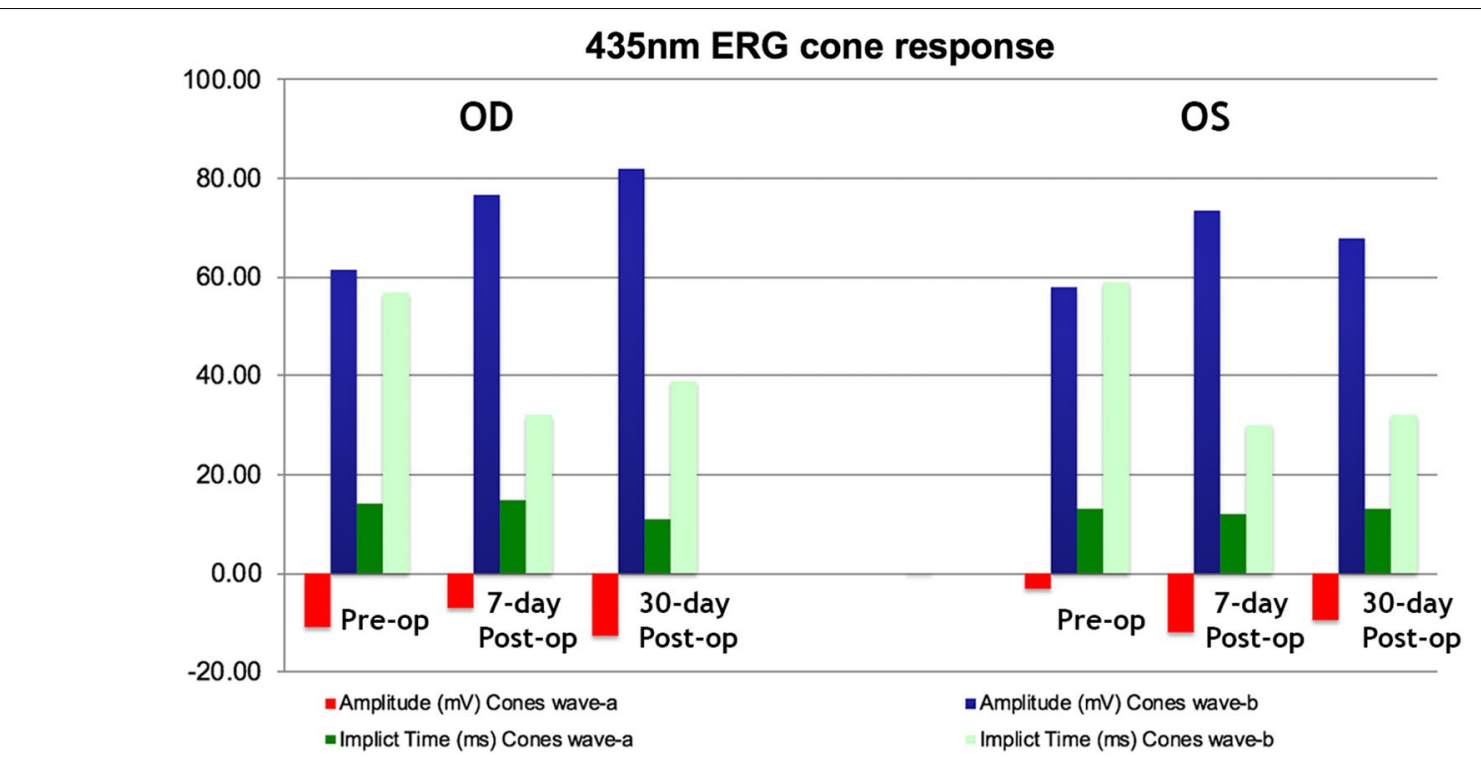

Fig. 11 Preoperative and postoperative ERG mean values from the animals exposed to $435 \mathrm{~nm}$ wavelength light without systemic administration of lutein. Diffuse reduction of the $a$ and b-wave amplitudes

In conclusion, the use of systemic administration of lutein showed no benefit to avoiding retinal phototoxicity generate to xenon light source using $435 \mathrm{~nm}$ and $420 \mathrm{~nm}$ filters.

\section{Abbreviations}

ARVO: Association for Research in Vision and Ophthalmology; ERG: electroretinography; FA: fluorescein angiography; ISCEV: International Society for Clinical Electrophysiology of Vision; MS: milliseconds; OCT: optical coherence tomography; RPE: retinal pigment epithelium; $\mu \mathrm{V}$ : microvolts.

\section{Authors' contributions}

AT-Conceptualizing the study, data acquisition, analyzing the data, interpreting the findings, writing \& reviewing the manuscript. EAN \& EB-Data acquisition and analysing the data. AL \& MEF-Interpreting the findings and reviewing the manuscript. RBJ_Analysing the data, interpreting the findings, reviewing the manuscript. All authors read and approved the final manuscript.

\section{Competing interests}

The authors declare that they have no competing interests. The authors also declare that this work was not presented in any meeting.

\section{Availability of data and materials}

The datasets used and/or analyzed during the current study are available from the corresponding author on reasonable request.

\section{Consent for publication \\ Not applicable.}

\section{Ethics approval and consent to participate}

This study was approved by the Federal University of São Paulo animal's ethics committee.

\section{Funding}

Grant information: This work was supported in part by CNPq (AT_Grant number 502530/2013-8).

\section{Publisher's Note}

Springer Nature remains neutral with regard to jurisdictional claims in published maps and institutional affiliations.

Received: 23 December 2018 Accepted: 27 March 2019

Published online: 07 May 2019

\section{References}

1. Aonuma $\mathrm{H}$, Yamazaki $\mathrm{R}$, Watanabe I. Retinal cell death by light damage. Jpn J Ophthalmol. 1999;43:171-9.

2. Herbert EN. Illumination and phototoxicity issues in vitreoretinal surgery. Retina. 2008;28:1175 author reply 1175

3. Ham WT Jr, et al. Action spectrum for retinal injury from near-ultraviolet radiation in the aphakic monkey. Am J Ophthalmol. 1982;93:299-306.

4. van den Biesen PR, et al. Endoillumination during vitrectomy and phototoxicity thresholds. Br J Ophthalmol. 2000;84:1372-5.

5. Yanagi Y, et al. Evaluation of the safety of xenon/bandpass light in vitrectomy using the A2E-laden RPE model. Graefes Arch Clin Exp Ophthalmol. 2007;245:677-81.

6. Roberts JE. Ocular phototoxicity. J Photochem Photobiol B. 2001;64:136-43.

7. Bone RA, Landrum JT, Tarsis SL. Preliminary identification of the human macular pigment. Vis Res. 1985;25:1531-5.

8. Bone RA, et al. Distribution of lutein and zeaxanthin stereoisomers in the human retina. Exp Eye Res. 1997;64:211-8.

9. Khachik F, Bernstein PS, Garland DL. Identification of lutein and zeaxanthin oxidation products in human and monkey retinas. Invest Ophthalmol Vis Sci. 1997;38:1802-11.

10. Snodderly DM, et al. The macular pigment. I. Absorbance spectra, localization, and discrimination from other yellow pigments in primate retinas. Investig Ophthalmol Vis Sci. 1984;25:660-73.

11. Snodderly DM, Auran JD, Delori FC. The macular pigment. II. Spatial distribution in primate retinas. Investig Ophthalmol Vis Sci. 1984;25:674-85.

12. Handelman GJ, et al. Carotenoids in the human macula and whole retina. Investig Ophthalmol Vis Sci. 1988;29:850-5.

13. Handelman GJ, et al. Measurement of carotenoids in human and monkey retinas. Methods Enzymol. 1992;213:220-30. 
14. Schalch W. Carotenoids in the retina - a review of their possible role in preventing or limiting damage caused by light and oxygen. EXS. 1992;62:280-98

15. Reading VM, Weale RA. Macular pigment and chromatic aberration. J Opt Soc Am. 1974;64:231-4.
16. Dogramaci $M$, et al. Foveal light exposure is increased at the time of removal of silicone oil with the potential for phototoxicity. Graefes Arch Clin Exp Ophthalmol. 2013;251:35-9.

17. De Vera Mudry MC, et al. Blinded by the light: retinal phototoxicity in the context of safety studies. Toxicol Pathol. 2013;41:813-25.
Ready to submit your research? Choose BMC and benefit from:

- fast, convenient online submission

- thorough peer review by experienced researchers in your field

- rapid publication on acceptance

- support for research data, including large and complex data types

- gold Open Access which fosters wider collaboration and increased citations

- maximum visibility for your research: over 100M website views per year

At BMC, research is always in progress.

Learn more biomedcentral.com/submissions 